\title{
Design and Development of a BlueGS Gateway for Bluetooth and GSM Protocols
}

\author{
Edgar Manuel Cano Cruz, Juan Gabriel Ruiz Ruiz, Luis Alberto Hernández Montiel
}

Computer Science Department, University of the Istmo Region, Ixtepec, Mexico

Email address:

ie.edgarcano@gmail.com (E. M. C. Cruz)

\section{To cite this article:}

Edgar Manuel Cano Cruz, Juan Gabriel Ruiz Ruiz, Luis Alberto Hernández Montiel. Design and Development of a BlueGS Gateway for Bluetooth and GSM Protocols. International Journal of Wireless Communications and Mobile Computing. Vol. 3, No. 6, 2015 , pp. 66-71. doi: $10.11648 /$ j.wcmc.20150306.12

\begin{abstract}
The new generation of mobile devices with embedded applications is one of the most rapidly growing technologies in the Wireless Networks. In this paper, we propose to combine the functions and capabilities of the maturity wireless protocols Bluetooth and GSM technologies, to design a powerful gateway tool for embedded systems: the BlueGS system. In addition, also included, is the construction of the BlueGS node, providing a flexible platform with the possibility of expanding the functionality. The system incorporates a Bluetooth and GSM module, thus achieving the development of a low cost system that does not require complex infrastructure to operate and is easily accessible to the general public.
\end{abstract}

Keywords: Security System, Embedded System, Motion Sensor, Bluetooth, GSM

\section{Introduction}

In the last two decade, many users have an interest in both Bluetooth and GSM technologies, because of their advantages such as, low-cost implementation and low energy consumption, which offer different advantages in the delivery of embedded systems, various types of devices have adopted wireless technologies such as, Wi-Fi, Bluetooth, and ZigBee, and have opened up new opportunities for new and innovative means of embedded systems delivery $[1,2]$. Consequently, these capabilities make these protocols a suitable wireless standard to build a platform with different levels of scalability, flexibility and easy to use adaptation on several projects.

The implementation of an embedded gateway system using wireless technologies, will in time, exploit the recent advances in WPAN, ubiquitous computing, wireless sensors and others areas $[3,4,5]$. The interoperability between Short-Range Wireless Protocols on embedded systems devices, can give to available to the user several functions that maximize the level of usability.

Bluetooth is clearly planned for short-range cable replacement for medium bandwidth device to device connections, and its most likely uses are ad hoc communication between mobile computing devices and fixed equipment.

Based on the extensive increase of applications on smart phones with Bluetooth, and in the analysis of the development of smart appliance devices with GSM technology in the industry [1], we propose combining both capabilities of these protocols for diagnostics, data transfer or configuration systems. Bluetooth and GSM are different by design and are optimized for different applications. In this way, the real industrial wireless networks will inevitably be hybrids, including Bluetooth and GSM, in complementary roles that suit the characteristics of each. The key to success will be in deploying the right wireless technologies for the requirements of the application and avoiding the temptation of trying to make one technology meet all needs.

This paper shows the design and implementation of a wireless embedded system gateway tool based on Bluetooth and GSM protocols. The rest of this paper is organized as follows: Section II introduces a brief related work; Section III of Short-Range Wireless Networks and presents the basics of Bluetooth and GSM wireless protocols; Section IV describes the design and implementation of the BlueGS system. In Section V, we present the preliminary results of the BlueGS system with a one basic application on a move sensor node. Finally, Section VI concludes this paper.

\section{Related Work}

In related work there are some emergent hybrid platforms to communicate devices between different wireless protocols. In 
the work [5], Galinina and colleagues envision a scenario where many in-home sensors are communicating with a smart gateway over the Bluetooth Low Energy protocol, while at the same time harvesting RF energy transmitted from the gateway wirelessly via a dedicated radio interface. The authors thoroughly investigate performance limitations of such wireless energy transfer interface (WETI) with dynamic analytical model and with important practical considerations. Their methodology delivers the upper bound on WETI operation coupled with BLE-based communication, which characterizes ultimate system performance over the class of practical radio and energy resource management algorithms.

Ruta et al. [6], presented a hybrid ZigBee/Bluetooth grid infrastructure to interconnect the user with interface nodes and to perform an advanced resource discovery. Li et al. [7], introduced a new method of conversion to achieve the ZigBee communication with CAN bus, realizing the link of CAN bus and the wireless network in order to conquer the conflictions brought from bus configuration and the protocol of controllers. Finally, Ren Xiaoghon et al. [8], gives a kind of design of CAN bus network based on Bluetooth technology, they indicated that the system could operate reliably and steadily and CAN-Bluetooth nodes could deliver the data correctly transmitted.

At work [8], Laine, Chaewoo and Haejung present a technical design of a Bluetooth-based mobile gateway that bridges the connection between a ZigBee sensor network and the Internet. Their system enables ubiquitous health care experience while providing a platform for additional services such as alarms, notifications and analysis of medical data. Controlling a sensor network from the mobile gateway is also possible. The flexible design of the system does not restrict its usage only to health care services - the gateway can be configured to work with any kind of sensor network having a sink node with Bluetooth capability.

\section{GSM and Bluetooth Network: A Brief Review}

\subsection{GSM}

According with [9], a GSM network has defined, the three subsystems involved are the mobile station subsystem, the base station subsystem, and the home subsystem (see Figure 1).

- The mobile station subsystem consists of the mobile equipment (ME) and a smart card called the Subscriber Identity Module (SIM). The mobile equipment is uniquely identified by the International Mobile Equipment Identity (IMEI). The SIM card contains the International Mobile Subscriber Identity (IMSI) used to identify the subscriber to the system, a secret key for authentication, and other information.

- The base station subsystem consists of the Base Transceiver Station (BTS) and the Base Station Controller (BSC). These are the connections between the mobile stations and the Mobile Switching Center (MSC).
- The home subsystem is composed of five parts, the Mobile Switching Center (MSC), the Home Location Register (HLR), the Visitor Location Register (VLR), the Authentication Center ( $\mathrm{AuC})$, and the Equipment Identity Register (EIR).

- The HLR is a database that stores complete local customer information. It is the main database. Your carrier puts your information on its nearest HRL, or the one assigned to your area. That info includes your IMEI, your directory number, and the class of service you have. It also includes your current city and your last known "location area" the place you last used your mobile.

- The VLR contains roamer information. Once the visited system detects your mobile, its VLR queries your assigned HLR. The VLR makes sure you are a valid subscriber, then retrieves just enough information from the now distant HLR to manage your call. It temporarily stores your last known location area, the power your mobile uses, special services you subscribe to and so on.

- The AuC stores a copy of the secret key kept in each subscriber's SIM card and generates authentication parameters for the authentication protocol on the request of HLRs.

- The EIR is a database that contains a list of all the valid mobile devices on the network, where each mobile station is identified by its International Mobile Equipment Identity (IMEI). The mobile stations communicate through radio links with the base stations, which are in turn connected to the MSC.

- The MSC is responsible for transiting signals between radio links and wire-lined networks.

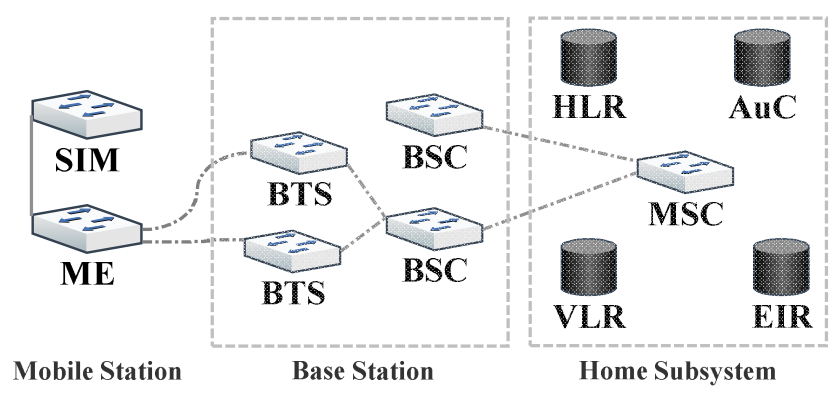

Figure 1. GSM network.

\subsection{Bluetooth}

Meanwhile, Bluetooth, also known as the IEEE 802.15.1 standard, is based on a wireless radio system designed for short-range and has cheap devices to replace cables [10]. The Figure 2 shown the Bluetooth topology that provides a point-to-point connection as well as a point-to-multipoint connection.

There are 79 Bluetooth channels in the $2.4 \mathrm{GHz}$ ISM band, each Bluetooth channel is divided into time slots and the duration of each time slot is $625 \mu \mathrm{s}$. Bluetooth devices can communicate with each other using the frequency hopping spread spectrum (FHSS). Bluetooth devices are divided into three power classes, where the maximum output power levels 
of classes 1,2 and 3 are set to $100 \mathrm{~mW}, 2.5 \mathrm{~mW}$, and $1 \mathrm{~mW}$ respectively [11].

The Bluetooth topology defines the basic network called piconet, and can form point-to-point (see Figure 2a) or point-to-multipoint links (see Figure $2 b$ ). The piconet is a set of two to eight Bluetooth devices. One device manages the transmission in each piconet, this device is called master, while the others devices are called slaves.
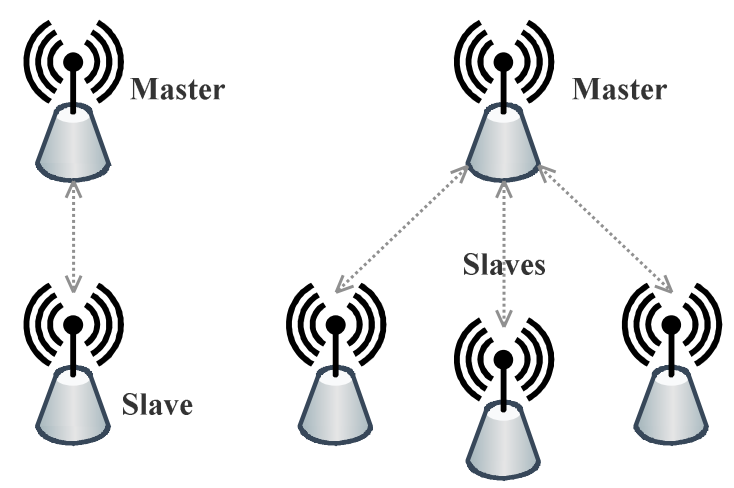

(a) Point-to-point (b) Point-to-multipoint

Figure 2. Bluetooth piconet.

\section{Design of the BlueGS System}

BlueGSM system implementation was done with the support of the methodology for the development of embedded systems SPIES [11], which is a specific methodology for modeling relationships between functionality, components, equipment and materials of an embedded system. SPIES consist of five phases describe at the follow (see Figure 3).

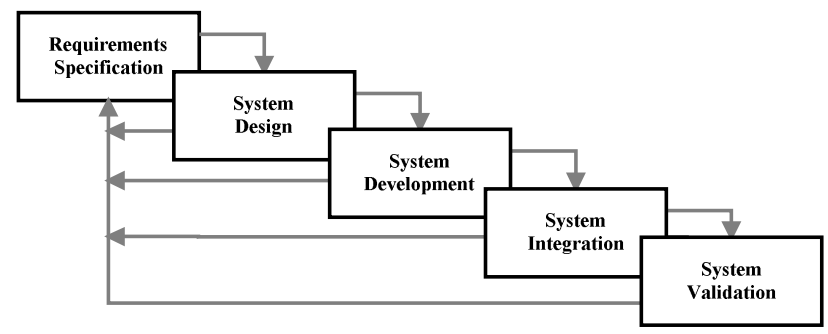

Figure 3. SPIES methodology.

\subsection{Requirements Specification}

The BlueGSM system is a gateway tool that converts data from Bluetooth devices to GSM data, and sends information through GSM network. The BlueGSM node (see Figure 4) consists of the follow components:

Arduino Uno is a microcontroller board based on the ATmega328 microcontroller [14]. This board has 14 digital pins of input / output (of which 6 can be used as PWM outputs), 6 analog inputs, a ceramic resonator of $16 \mathrm{MHz}$, a USB connection, a power jack an ICSP header, and a button reset. It contains everything needed to support the microcontroller.

Through the DTE module the user configures the node and displays the messages that are sending between devices. Within the DTE module will find the following components.

The DCE modules are responsible for establishing the different connections according to the protocol used and for transmitting or receiving information in serial format.

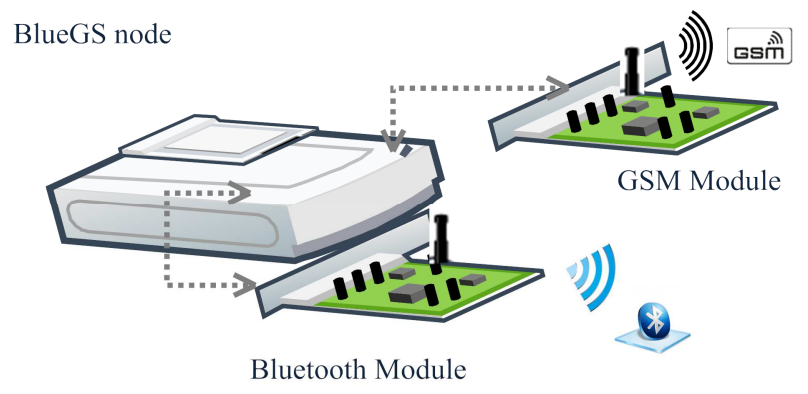

Figure 4. Gateway System.

The DCE module consists of follow components:

Bluetooth Hc-05The HC-05 module is a Bluetooth device that is configured at the factory to work as master or slave. In the master mode you can connect with other Bluetooth modules, while in the slave mode is listening to connection requests. This module allows remote control from a cell phone or a laptop all the functionality desired in a system.

The BlueGS node operates in two main modes: Configuration Mode and Data Mode (see Figure 5). These operating main modes are managed by the Operation Management subsystem. The BlueGS node starts up in Data Mode and the user can be requested to move to Configuration Mode by sending an escape sequence through the module DTE. Although in some situations it is necessary to restore the BlueGS node settings to their default values.

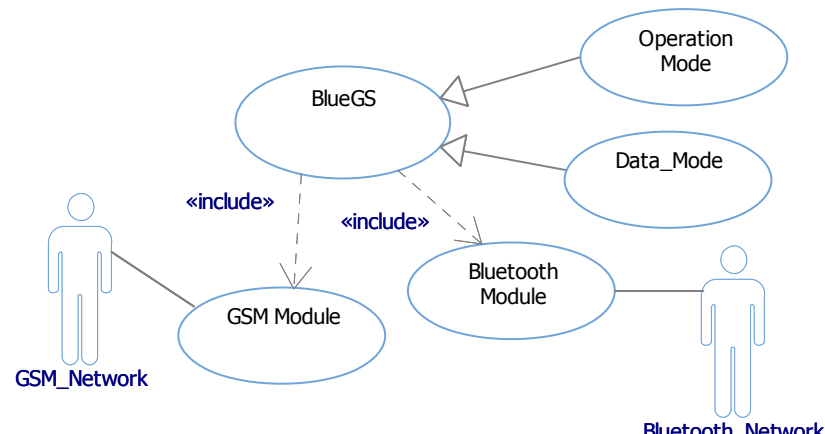

Figure 5. Use case diagram for BlueGS node.

\subsection{System Design}

In the BlueGS Configuration Mode the user set up both GSM and Bluetooth modules, through the Bluetooth Configuration Management and GSM Configuration Management subsystems. A Bluetooth module can be in several different operation modes, which determines whether or not the BlueGS node can be connected to and whether or not other Bluetooth devices performing searches can discover a BlueGS node. Serial connections are Bluetooth connections 
based on the Serial Port Profile (SPP). In this sense, it is important that devices, the BlueGS node and Bluetooth device have the same profile to establish the link and can communicating between them.

Into the functionality of the BlueGS system, there exist tow subsystem to manage all operation of the gateway: Bluetooth Configuration Management and GSM Configuration Management.

In the Bluetooth Configuration Management, the Bluetooth Mode enable or disable the Bluetooth characteristic of the BlueGS node and if can work in discoverable and non-discoverable mode. The Name Mode is useful to change the friendly name of the Bluetooth Module into the BlueGS node. Finally, the Pairing Mode consists of four methods to search a Bluetooth device and establish a security link connection with the BlueGS node.

In the GSM Configuration Management, to enable the GSM module and establish a real cell phone number where the data information will receive, the GSM module is configured.

In the BlueGS Data Mode, the system can manage both Bluetooth and GSM, and convert Bluetooth data in GSM data, but not in vice versa mode. Within the Data Management subsystem, the BlueGS can send and receive Bluetooth and GSM data, but only one packet at time, also is necessary that in a previously steps, the user active and configure the Bluetooth and GSM modules and change the operation mode of the BlueGS node to data mode to establish connection with the respective devices.

Once that the modules are configured, is possible that the BlueGS convert GSM packets to Bluetooth packets. When the BlueGS node is in the Converting Data Mode subsystem, the BlueGS node can listen and receive packets from GSM network and convert to Bluetooth data, and then send data to other Bluetooth device (both devices have been previously paired).

\subsection{System Development}

To prepare the project, we have considered a maximum budget of $\$ 50.00$ USD, considering the features that are contemplated in the system, the table 1 shows the tools currently in the system.

Table 1. Costs of the tools [12].

\begin{tabular}{ll}
\hline Tool & Cost (USD) \\
\hline 1 Arduino Mega 2560 & $\$ 24.95$ \\
1 Bluetooth Hc-05 & $\$ 3.90$ \\
1 GPRS SIM800L & $\$ 13.83$ \\
Arduino IDE & $\$ 0.00$ \\
Android Studio & $\$ 0.00$ \\
Total & $\$ 42.68$ \\
\hline
\end{tabular}

\subsubsection{Arduino Uno}

Arduino Uno is a microcontroller board based on the ATmega328 microcontroller [13]. This board has 14 digital pins of input / output (of which 6 can be used as PWM outputs), 6 analog inputs, a ceramic resonator of $16 \mathrm{MHz}$, a
USB connection, a power jack an ICSP header, and a button reset (see Figure 6). It contains everything needed to support the microcontroller.

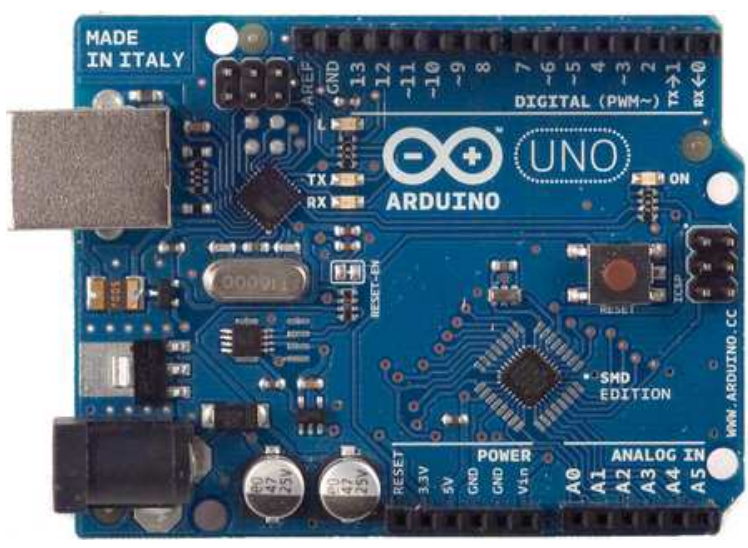

Figure 6. Arduino Uno.

\subsubsection{Bluetooth $\mathrm{Hc}-05$}

The HC-05 module is a Bluetooth device that is configured at the factory to work as master or slave (see Figure 7). In the master mode you can connect with other Bluetooth modules, while in the slave mode is listening to connection requests. This module allows remote control from a cell phone or a laptop all the functionality desired in a system.

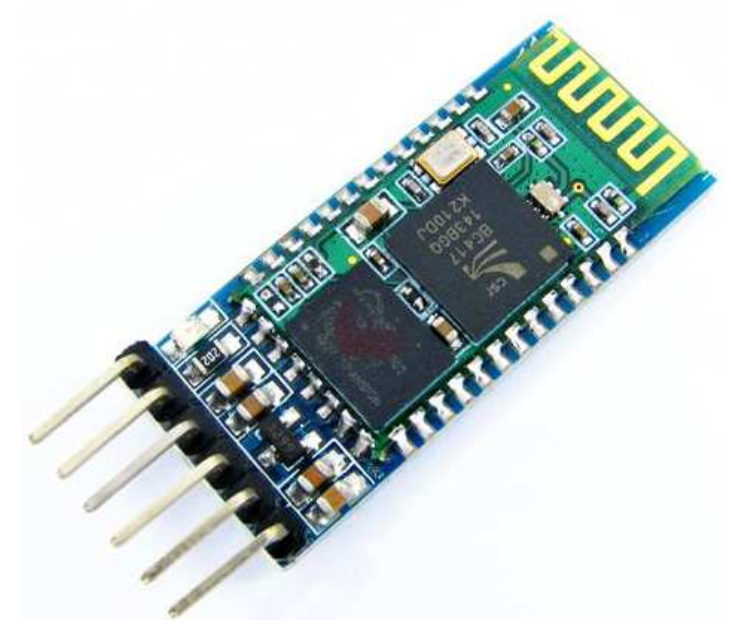

Figure 7. Bluetooth Hc-05 wireless module.

\subsubsection{GPRS SIM800L}

The GPRS module based on SIM800L, supports quad-band GSM/GPRS network, available for GPRS and SMS message data remote transmission. The GSM module can be used with a direct link to the microcontroller through its TTL serial port. No need MAX232. Power on the module automatically boot automatically search network. Onboard signal lights (with signal flash slowly, no signal flash quickly).

\section{Results}

A partial integration of BlueGS was performed, and test the operation of a move sensor. In the Figure 8 the plan 
implemented between the move sensor and the Arduino board is shown.

The system was validated through a monitoring application with the move sensor running on the Arduino board and an application on a smartphone with Android operating system.
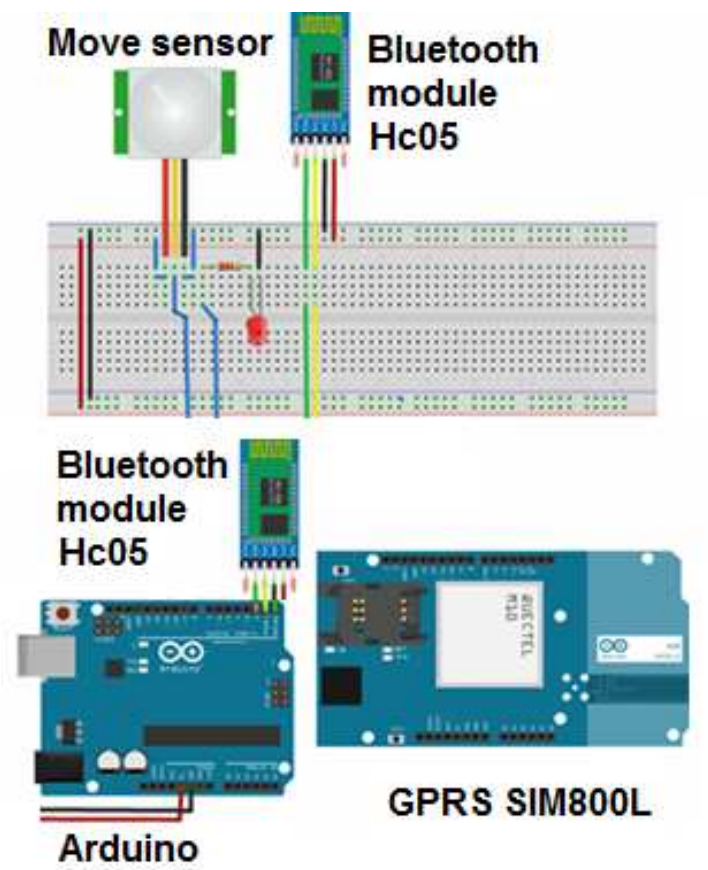

Figure. 8. Scheme system for validation.

System operation is very simple, the move sensor is connected at Arduino board through Bluetooth connection (see Figure 9), if the move sensor detects movement, and then the android device will process the data and send a text message to the number that was established.
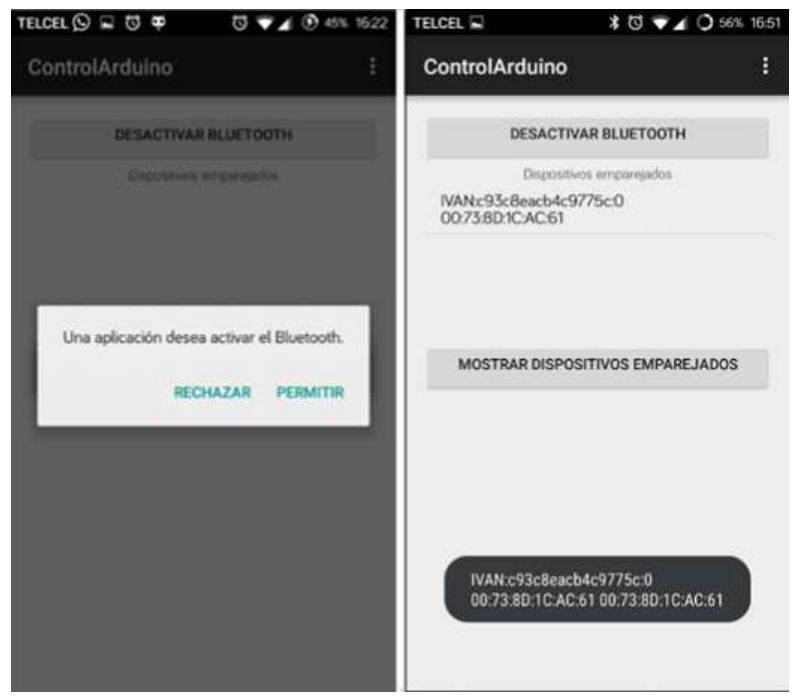

Figure 9. Management of the Bluetooth connection.

\section{Conclusions}

The development of a Bluetooth and GSM gateway system is proposed in this paper. The system validation consisted of the move sensor that is detect measures variables whereas Bluetooth protocol establishes the communication between sensors and the Arduino system where this parameters trigger a gsm module to send a sms text to the number that was previously established.

In future research, we hope to develop the weather system by using the ARDUINO Mega card to replace the current card and provide in this form more functionalities to the BlueGS gateway system. The fusion of both wireless technologies Bluetooth and GSM, made it achievable to delivery sensor networks, industrial control systems, location and position throughout mobile users environments, in order to capture different types of data information. Also, using the capabilities of these wireless protocols, it gives different solutions such as low-cost and low energy consumption to delivery and build embedded systems.

\section{References}

[1] Wang, Q., Rackers, J. A., He, C., Qi, R., Narth, C., Lagardere, L., \& Ren, P. (2015). A General Model for Treating Short-Range Electrostatic Penetration in a Molecular Mechanics Force Field. Journal of Chemical Theory and Computation.

[2] Bazydło, P., Dąbrowski, S., \& Szewczyk, R. (2015). Wireless temperature measurement system based on the IQRF platform. In Mechatronics-Ideas for Industrial Application (pp. 281-288). Springer International Publishing.

[3] Kaur Kapoor, N., Majumdar, S., \& Nandy, B. (2015). Techniques for Allocation of Sensors in Shared Wireless Sensor Networks. Journal of Networks, 10(01), 15-28.

[4] De Souza, R. H., Savazzi, S., \& Becker, L. B. (2015). Network design and planning of wireless embedded systems for industrial automation. Design Automation for Embedded Systems, 1-22.

[5] Galinina, O., Mikhaylov, K., Andreev, S., Turlikov, A., \& Koucheryavy, Y. (2015). Smart home gateway system over Bluetooth low energy with wireless energy transfer capability. In EURASIP Journal on Wireless Communications and Networking. Springer International Publishing.

[6] Ruta, M., Scioscia, F. Di Noia, T., \& Di Sciascio, E. A hybrid ZigBee/Bluetooth approach to mobile semantic grids, International Journal of Computer Systems Science and Engineering (IJCSSE) - Special issue on Mobile Data Management: Models, Methodologies and Services, 2009.

[7] Y. Li, L. Yu, J. Yan, and H. Li, "The design of Zig Bee communication convertor based on CAN," International Conference on Computer Application and System Modeling (ICCASM), IEEE Computer Society, 245-249, 2010.

[8] R. Xiaohong, F. Chenghua, W. Tianwen, and J. Shuxiang, "CAN Bus Network Design Based on Bluetooth Technology," Electrical and Control Engineering (ICECE), 2010 International Conference on, vol., no., pp.560-564, 25-27 June 2010 .

[9] GSMA, «GSMA,» 0203 2015. [En línea]. Available: http://www.gsma.com/aboutus/gsm-technology/gsm. 
[10] Bluetooth SIG, "Bluetooth Specification v4.0", 2009.

[11] Garcia. I, and Cano. E. Designing and implementing a constructionist approach for improving the teaching-learning process in the embedded systems and wireless communications areas, Computer Applications in Engineering Education: Wiley Periodicals, Inc. 2011.
[12] Arduino, «The community of Arduino,» 2015. [En línea]. Available: http://www.arduino.cc/. [Accessed May 2015].

[13] Electrodragon, "Electrodragon," 2015. [Online]. Available: $\mathrm{http}: / /$ www.electrodragon.com/. [Accessed 2015]. 